\title{
Analysis of Internet Traffic Distribution for User Behaviour based Probability in Two-Market Environment
}

\author{
Virendra Kumar Tiwari \\ Associate Professor, \\ Deptt of MCA, \\ Lakshmi Narain College of \\ Technology (LNCT), Bhopal \\ (M.P.), 462021, India
}

\author{
Sanjay Thakur \\ Professor \& HoD,Department \\ of Computer Science, Lord \\ Krishna College of Technology, \\ Indore, India.
}

\author{
D. Shukla \\ Deptt. of Math. and Statistics, \\ Dr. H.S. Gour University, \\ Sagar (M.P.), 470003, India
}

\begin{abstract}
Consider two markets and two operators having different networks operators. Both operators are in competition for capturing more and more the internet traffic. The users have presumptive behaviour like faithful, impatient and completely impatient. This paper presented Markov chain model based analysis of user behaviour for selecting any one operator. It is found that blocking probability of network plays important role for determining the user's behaviour towards choosing an operator as internet service provider. Also it contains analysis of initial share over the blocking probability varying probability of the rest-state.
\end{abstract}

Keywords: Markov chain model, Blocking probability, Callby-call basis, Internet traffic, Quality of Service (QoS), Users behavior.

\section{INTRODUCTION}

Markov Chain Model is a technique of exploring the transition behavior of a system. Naldi [13] has opened up the problem of internet traffic sharing evaluation. Shukla, Gadewal [6] have shown the application of Markov Chain model to the modelling of space division switches. Shukla, Pathak and Thakur [7] have predicated useful contribution for modelling of internet traffic sharing phenomena between two operators in competitive markets. Vern Paxson [17] has discussed the experiences with different measurement and analysis of the Internet Traffic. Hambali and Ramani [3] have proposes a new architecture of the switch for the demand of multicast service and cell loss with delay will decrease the performance of an ATM networks. Hemangi and Murali et. al. [2] has presenting a new protocol for probabilistic quality of service analysis for distributed control system. Al-Shargabi and Ismail [15] have proposes internet compressed traffic: a solution for the explosion of the internet.

Shukla, Tiwari, Thakur and Deshmukh [18] have given a share loss analysis of internet traffic distribution in computer networks. Andrikopoulos et al. [8] have providing rate guarantees for internet application traffic across ATM networks. Medhi [11],[12] contains the foundational aspects of Markov chains in the context of stochastic processes. Dorea and Rajas [5] have shown the application of Markov chain models in data analysis. Aggarwal and Kaur [16] have proposed reliability analysis of fault-tolerant in a multistage interconnection on computer networks. Shukla, Tiwari and Thakur [21] have shown the effects of disconnectivity analysis for congestion control in internet traffic sharing. Yuan and lygevers [4] obtained the stochastic differential equations and proved the criteria of stabilization for Mrakovian switching.

Shukla, Tiwari et. al. [19],[20] have discussed a comparison of methods for internet traffic sharing in computer network. Newby and Dagg [14] presented a maintenance policy for stochastically deteriorating systems, with the average cost criteria. Shukla and Thakur [9] have useful contribution on the use of crime based user analysis in Internet traffic sharing under cyber crime. Mohammed and Ramli et.al [1] has analyses of an authentication protocol for mobile cellular network. Some other useful similar contributions are due to Perzen[10].

\section{USER'S BEHAVIOR AND MARKOV CHAIN MODEL}

Let $O_{i}$ and $O_{j}(i=1,3 ; j=2,4)$ be operators (or ISP) in two competitive locations Market-I and Market-II. Users choose first to a market and then enters into cyber cafe (or shop) situated in that market where computer terminals for specific operators are available to access the Internet. Let $\left\{X^{(n)}, n \geq 0\right\}$ be a Markov chain having transitions over the state space $O_{1}, O_{2}, O_{3}$, $O_{4}, R_{1}, R_{2}, Z_{1}, Z_{2}, A, M_{1} \& M_{2}$ where

State $O_{1}$ : first operator in market-I

State $\mathrm{O}_{2}$ : second operator in market-I

State $\mathrm{O}_{3}$ : third operator in market-II

State $\mathrm{O}_{4}$ : fourth operator in market-II

State $R_{1}$ : temporary short time rest in market-I

State $R_{2}$ : temporary short time rest in market-II

State $Z_{l}$ : success (in connectivity) in market-I

State $Z_{2}$ : success (in connectivity) in market-II

State $A$ : abandon to call attempt process

State $M_{1}$ : market-I

State $M_{2}:$ market-II 
The $X^{(n)}$ stands for state of random variable $X$ at $n^{\text {th }}$ attempt $(n \geq 0)$ made by a user. Some underlying assumptions of the model are:

a) User first selects the Market-I with probability $q$ and Market-II with probability $(1-q)$ as per ease.

b) After that user, in a shop, chooses the first operator $O_{i}$ with probability $p$ or to next $O_{j}$ with $(1-p)$.

c) The blocking probability experienced by $O_{i}$ is $L_{l}$ and by $O_{j}$ is $L_{2}$.

d) Connectivity attempts of user between operators are on call-by-call basis, which means if the call for $O_{i}$ is blocked in $k^{\text {th }}$ attempt $(k>0)$ then in $(k+1)^{\text {th }}$ user shifts to $O_{j}$. If this also fails, user switches to $O_{i}$ in $(k+2)^{t h}$.

e) Whenever call connects through either $O_{i}$ or $O_{j}$ we say system reaches to the state of success $\left(Z_{1}, Z_{2}\right)$.

f) The user can terminate call attempt process, marked as system to abandon state $A$ with probability $P_{A}$ (either from $O_{i}$ or from $O_{j}$ ).

g) If user reaches to rest state $R_{k}(k=1,2)$ from $O_{i}$ or $O_{j}$ then in next attempt he may either with a call on $O_{i}$ or $O_{j}$ with probability $r_{k}$ and $\left(1-r_{k}\right)$ respectively.

h) From state $R_{k}$ user cannot move to states $Z_{k}$ and $A$.

The transition diagram is in figure 1 to explain the details of assumptions and symbols. In further discussion, operator $O_{1}=O_{3}$ and $O_{2}=O_{4}$ is assumed with network blocking parameter $L_{1}=L_{3}$, $L_{2}=L_{4}$.

\section{LOGIC FOR TRANSITION PROBABILITY IN MODEL}

(a) The starting conditions ( state distribution before the first call attempt) are

$$
\begin{aligned}
& P\left[X^{(0)}=O_{1}\right]=0, \quad P\left[X^{(0)}=R_{1}\right]=0, \\
& P\left[X^{(0)}=O_{2}\right]=0, \quad P\left[X^{(0)}=R_{2}\right]=0, \\
& P\left[X^{(0)}=Z\right]=0 \text {, } \\
& P\left[X^{(0)}=A\right]=0, \\
& P\left[X^{(0)}=M_{1}\right]=q, \\
& P\left[X^{(0)}=M_{2}\right]=1-q
\end{aligned}
$$

(b) If in $(n-1)^{\text {th }}$ attempt, call for $O_{i}$ is blocked, the user may abandon the process in the $n^{\text {th }}$ attempts.

$P\left[X^{(n)}=A / X^{(n-1)}=O_{i}\right]=P\left[\right.$ blocked at $\left.O_{i}\right] \cdot P[$ abandon the process $]=L_{i} P_{A}$

Similar for $O_{j}$,

$P\left[X^{(n)}=A / X^{(n-1)}=O_{j}\right]=P\left[\right.$ blocked at $\left.O_{j}\right] \cdot P[$ abandon the process $]=L_{j} P_{A}$

(c) At $O_{i}$ in $n^{\text {th }}$ attempts call may be made successfully and system reaches to state $Z_{k}$ from $O_{i}$. This happens only when call does not block in $(n-1)^{\text {th }}$ attempt

$$
P\left[X^{(n)}=Z_{k} / X^{(n-1)}=O_{i}\right]=P\left[\text { does not blocked at } O_{i}\right]=\left(1-L_{i}\right)
$$

Similar for $O_{j}$,

$$
P\left[X^{(n)}=Z_{k} / X^{(n-1)}=O_{j}\right]=P\left[\text { does not blocked at } O_{j}\right]=\left(1-L_{j}\right)
$$

(d) If user is blocked at $O_{i}$ in $(n-1)^{\text {th }}$ attempts, does not want to abandon, then in $n^{\text {th }}$ he shifts to operator $O_{j}$.

$P\left[X^{(n)}=O_{j} / X^{(n-1)}=O_{i}\right]=P\left[\right.$ blocked at $\left.O_{i}\right] \cdot P[$ does not abandon $]=L_{i}\left(1-p_{A}\right)$.

Similar for $O_{j}$,

$P\left[X^{(n)}=O_{i} / X^{(n-1)}=O_{j}\right]=P\left[\right.$ blocked at $\left.O_{i}\right] \cdot P[$ does not abandon $]=L_{j}\left(1-p_{A}\right)$

(e) For operator $O_{i} \cdot P\left[X^{(n)}=O_{i} / X^{(n-1)}=R_{k}\right]=r_{k}$.

Similar for $O_{j}, P\left[X^{(n)}=O_{j} / X^{(n-1)}=R_{k}\right]=1-r_{k}$.

(f) For $M_{k},(k=1,2)$ for $O_{i}, O_{j}$ $P\left[X^{(n)}=O_{i} / X^{(n-1)}=M_{k}\right]=p$.

Similar for $O_{j}$,

$$
P\left[X^{(n)}=O_{j} / X^{(n-1)}=M_{k}\right]=1-p .
$$

\section{CATEGORIES OF USERS}

Define three types of users as

(i) Faithful User (FU).

(ii) Partially Impatient User (PIU).

(iii) Completely Impatient User (CIU).

\section{SOME RESULTS FOR $\boldsymbol{n}^{\text {th }}$ ATTEMPTS}

At $n^{\text {th }}$ attempt, the probability of resulting state is derived in following theorems for all $n=0,1,2,3,4,5 \ldots$ for market-I.

$$
\begin{aligned}
& A=\left[L_{1}\left(1-P_{A}\right) P_{R_{1}} r_{1}\right], B=\left[L_{2}\left(1-P_{A}\right) P_{R_{1}}\left(1-r_{1}\right)\right], \\
& C=\left[L_{1} L_{2}\left(1-P_{A}\right)^{2}\left(1-P_{R_{1}}\right)^{2}\right], D=\left[L_{1}^{2} L_{2}\left(1-P_{A}\right)^{3}\left(1-P_{R_{1}}\right)^{2} P_{R_{1}}\right], \\
& E=\left[L_{2}^{2}\left(1-P_{A}\right)^{2}\left(1-P_{R_{1}}\right) P_{R_{1}}\right]
\end{aligned}
$$

Theorem 5.1: If user is FU and restrict to only $O_{l}$ and $R_{l}$ in $M_{l}$ then $n^{\text {th }}$ step transitions probability is

$$
\begin{aligned}
& P\left[X^{(2 n)}=O_{1}\right]=p A^{n} \\
& P\left[X^{(2 n+1)}=O_{1}\right]=q p A^{n}
\end{aligned}
$$

Theorem 5.2: If user is FU and restrict to only $O_{2}$ and $R_{I}$ then $n^{\text {th }}$ step transitions probability is

$$
\begin{aligned}
& P\left[X^{(2 n)}=O_{2}\right]=(1-p) B^{n} \\
& P\left[X^{(2 n+1)}=O_{2}\right]=q(1-p) B^{n}
\end{aligned}
$$




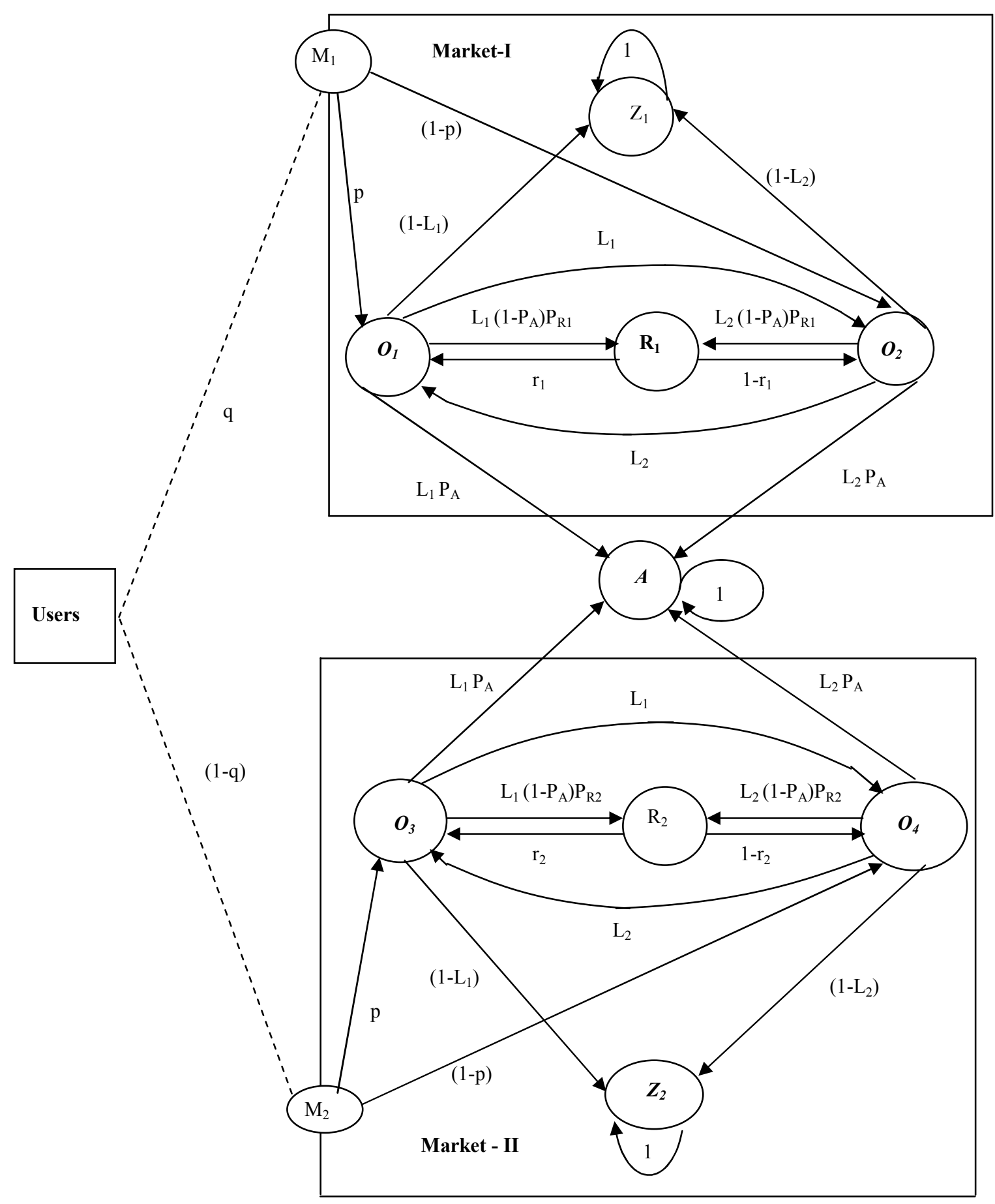

Fig 1: Transition Diagram of Model 


\section{THE TRANSITION PROBABILITY MATRIX}

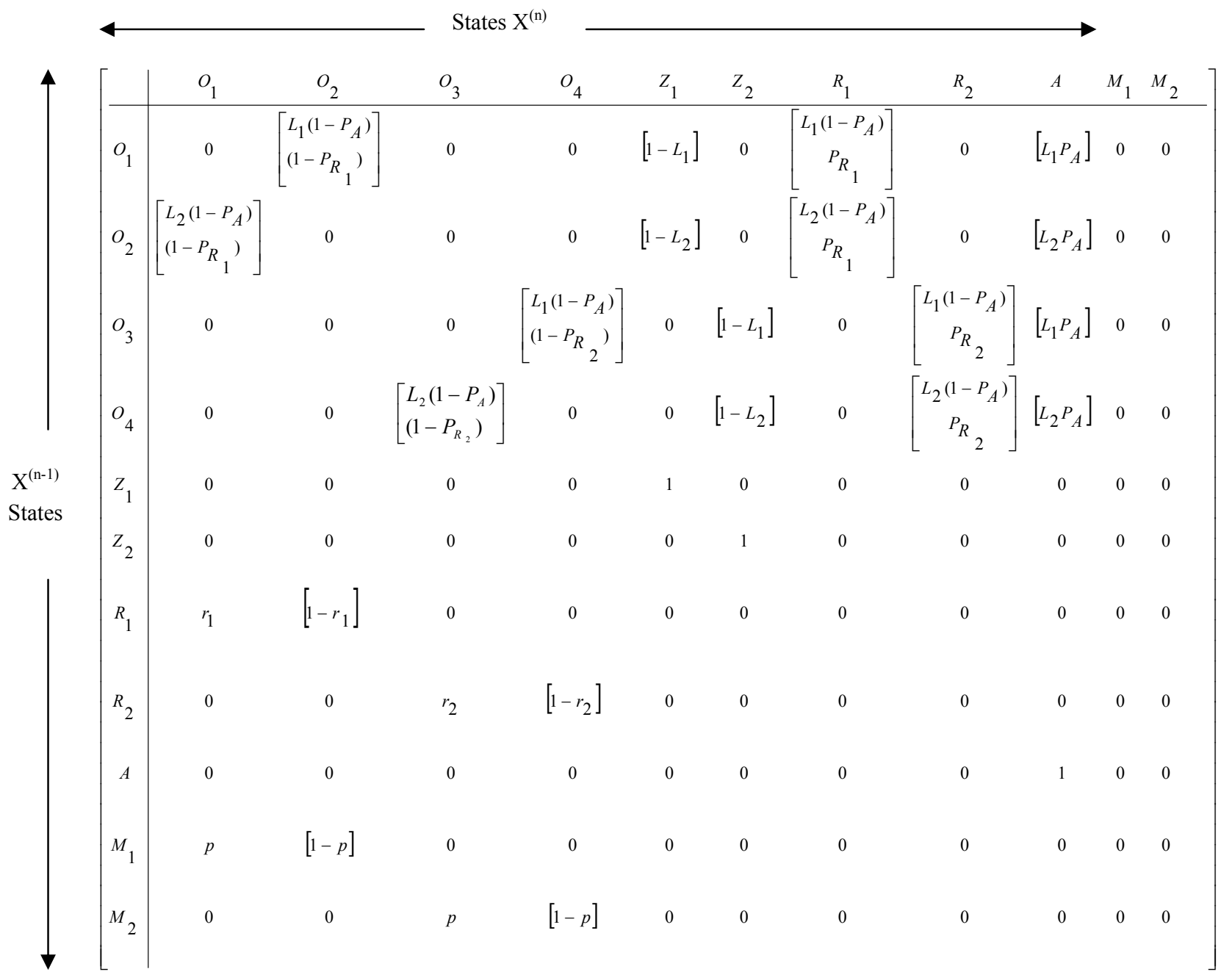

Fig 2: Transition Probability Matrix.

Theorem 5.3: If user is PIU and restricts to attempt between $O_{1}$ and $O_{2}$ and not interested to state $R$ in $M_{1}$ then

$$
\begin{aligned}
& P\left[X^{(2 n)}=O_{1}\right]=\frac{\left[q(1-p) C^{(n)}\right]}{\left[L_{1}\left(1-p_{A}\right)\left(1-p_{R_{1}}\right)\right]} \\
& P\left[X^{(2 n+1)}=O_{1}\right]=\left[q p C^{(n)}\right] \\
& \left.P\left[X^{(2 n)}=O_{2}\right]=\frac{\left[q p C^{(n)}\right]}{\left[L_{2}\left(1-p_{A}\right)\left(1-p_{R_{1}}\right)\right.}\right] \\
& P\left[X^{(2 n+1)}=O_{2}\right]=\left[q(1-p) C^{(n)}\right]
\end{aligned}
$$

Theorem 5.4: If user is CIU and attempts among $O_{1}, O_{2}$ and $R$ only in $M_{1}$ then at $n^{\text {th }}$ attempt the approximate probability expression are

$$
\begin{aligned}
P\left[X^{(2 n)}=O_{1}\right]= & {\left[\frac{\left[q(1-p) C^{(n)}\right]}{\left[L_{1}\left(1-p_{A}\right)\left(1-p_{R_{1}}\right)\right]}\right.} \\
+ & {\left[\frac{\left[p C^{(n)} p_{R_{1}} r_{1}\right]}{\left[L_{2}\left(1-p_{A}\right)\left(1-p_{R_{1}}\right)^{2}\right]}\right.} \\
P\left[X^{(2 n+1)}=O_{1}\right] & =\left[q p C^{n}\right] \\
& +\frac{\left[(1-p) C^{(n)} L_{2} p_{R_{1}}\left(1-r_{1}\right)\right]}{\left[L_{1}\left(1-p_{R_{1}}\right)\right]}
\end{aligned}
$$




$$
\begin{aligned}
P\left[X^{(2 n)}=O_{2}\right]= & {\left[\frac{\left[q p C C^{(n)}\right]}{\left[L_{2}\left(1-p_{A}\right)\left(1-p_{R_{1}}\right)\right]}\right.} \\
+ & {\left[(1-p) C^{(n)} p_{R_{1}}\left(1-r_{1}\right)\right] } \\
P\left[X^{(2 n+1)}=O_{2}\right]= & {\left[q(1-p) C^{(n)}\right] } \\
& +\frac{\left[p C_{R_{1}}^{(n)} L_{1} p_{R_{1}} r_{1}\right]}{\left[L_{1}\left(1-p_{A}\right)\right]} \\
& {\left[L_{2}\left(1-p_{R_{1}}\right)\right] }
\end{aligned}
$$

\section{BEHAVIOR OVER LARGE NUMBER OF ATTEMPTS FOR TRAFFIC SHARING}

Suppose $n$ is very large, then $\overline{P_{k}}=\left[\lim _{n \rightarrow \infty}{\overline{P_{k}}}^{(n)}\right], k=1,2$ and we get final traffic shares,

$$
\begin{aligned}
& {\left[\bar{P}_{1}\right]_{F U}=\left\{\frac{\left(1-L_{1}\right) \cdot p}{1-\left[A^{2}\right]}\right\}+\left\{\frac{\left(1-L_{1}\right) \cdot q p[A]}{1-\left[A^{2}\right]}\right\}} \\
& {\left[\bar{P}_{2}\right]_{F U}=\left\{\frac{\left(1-L_{2}\right) \cdot(1-p)}{1-\left[B^{2}\right]}\right\}+\left\{\frac{\left(1-L_{2}\right) \cdot q(1-p)[B]}{1-\left[B^{2}\right]}\right\}} \\
& {\left[\bar{P}_{1}\right]_{P I U}=\left\{\left(1-L_{1}\right) \cdot p+\frac{\left(1-L_{1}\right) \cdot p q[C]}{1-\left[C^{2}\right]}\right\}+\left\{\frac{\left(1-L_{1}\right) \cdot q p[C]}{1-\left[C^{2}\right]}\right\}}
\end{aligned}
$$$$
\left\lfloor\overline{P_{2}}\right\rfloor_{P I U}=\left(1-L_{2}\right)(1-p)
$$$$
+\left\{\frac{\left(1-L_{2}\right)(1-p) q[C]}{1-\left[C^{2}\right]}\right\}+\left\{\frac{\left(1-L_{2}\right) \cdot q(1-p)[C]}{1-\left[C^{2}\right]}\right\}
$$$$
\left[\bar{P}_{1}\right]_{C I U}=\left(1-L_{1}\right) p\left\{1+\left[\frac{q[C]}{1-\left[C^{2}\right]}\right]+\left[\frac{\left[D r_{1}\right]}{1-\left[C^{2}\right]}\right]\right\}
$$$$
+\left\{\left[\frac{q\left(1-L_{1}\right) L_{2}\left(1-P_{A}\right)\left(1-P_{R_{1}}\right)[C]}{1-\left[C^{2}\right]}\right]+\left[\frac{\left(1-L_{1}\right)\left(1-r_{1}\right)[E]}{1-\left[C^{2}\right]}\right]\right\}
$$$$
\left[\overline{P_{2}}\right]_{C I U}=\left(1-L_{2}\right) p\left\{1+\left[\frac{q[C]}{1-\left[C^{2}\right]}\right]+\left[\frac{\left[D\left(1-r_{1}\right)\right]}{1-\left[C^{2}\right]}\right]\right\}
$$$$
+\left\{\left[\frac{q\left(1-L_{2}\right) L_{2}\left(1-P_{A}\right)\left(1-P_{R_{1}}\right)[C]}{1-\left[C^{2}\right]}\right]+\left[\frac{\left(1-L_{2}\right) r_{1}[E]}{1-\left[C^{2}\right]}\right]\right\}
$$

\section{AVERAGE BLOCKING PROBABILITY EXPERIENCE BY USERS}

The user experiences varying average blocking probability, at $n^{\text {th }}$ attempt, described as:

$$
B_{i}^{(n)}=\frac{P\left[X^{(n-1)}=O_{1}\right] L_{1}+P\left[X^{(n-1)}=O_{2}\right] L_{2}}{P\left[X^{(n-1)}=O_{1}\right]+P\left[X^{(n-1)}=O_{2}\right]}
$$

[See Naldi (2002)]

In case of faithful user, by using theorem 5.1 and 5.2.

$\left\lfloor B_{i}^{(n)}\right\rfloor_{F U}=p \mathrm{~L}_{1}+(1-p) \mathrm{L}_{2} \quad[$ See Naldi (2002)]

For Partially Impatient User (PIU), using theorem 5.3

$$
\begin{array}{ll}
{\left[B_{i}{ }^{(n)}\right]_{P I U}} & =\frac{1}{p L_{1}+(1-p) L_{2}} \quad \text { for } n \text { even } \\
\left\lfloor B_{i}^{(n)}\right\rfloor_{P I U}=p L_{1}+(1-p) L_{2} & \text { for } n \text { odd }
\end{array}
$$

\section{COMPARISONS AMONG USERS}

(a)

$$
\begin{aligned}
{\left[B i^{(n)}\right]_{P I U} } & <\left[B i^{(n)}\right]_{F U} \\
\text { when } \mathrm{p} & <1 \text { along with } \mathrm{L}_{1}>\mathrm{L}_{2} \\
{\left[B i^{(n)}\right]_{C I U} } & <\left[B i^{(n)}\right]_{F U} \\
\text { when } \mathrm{p} & <1 \text { along with } \mathrm{L}_{1}>\mathrm{L}_{2} \\
{\left[B i^{(n)}\right]_{C I U} } & <\left[B i^{(n)}\right]_{P I U} \\
\text { when } \mathrm{p} & <1 \text { along with } \mathrm{L}_{1}>\mathrm{L}_{2}
\end{aligned}
$$$$
\text { (b) } \quad\left[B i^{(n)}\right]_{C I U}<\left[B i^{(n)}\right]_{F U}
$$$$
\text { (c) } \quad\left[B i^{(n)}\right\rfloor_{C I U}<\left\lfloor B i^{(n)}\right\rfloor_{P I U}
$$

\section{INITIAL TRAFFIC SHARE ANALYSIS}

According to figure 3 - 8 with the increase of blocking probability of operator $O_{1}$ the initial traffic share depends highly on opponents blocking probability $L_{2}$. If $L_{2}$ is high the initial traffic share of faithful users of $O_{I}$ is high.

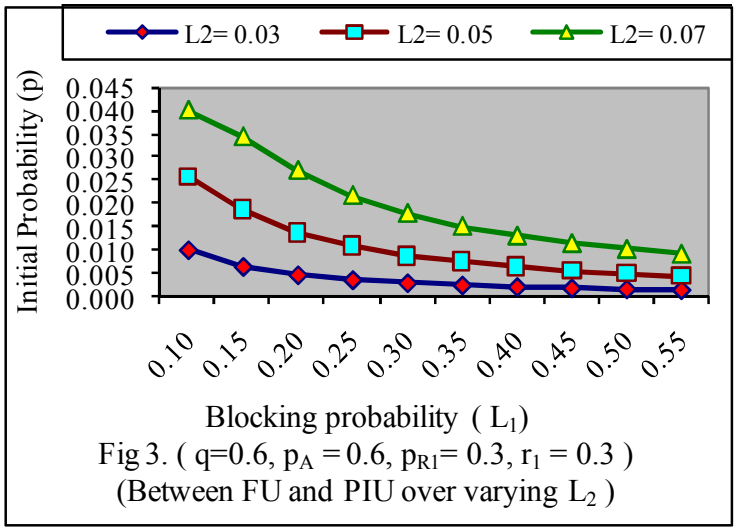




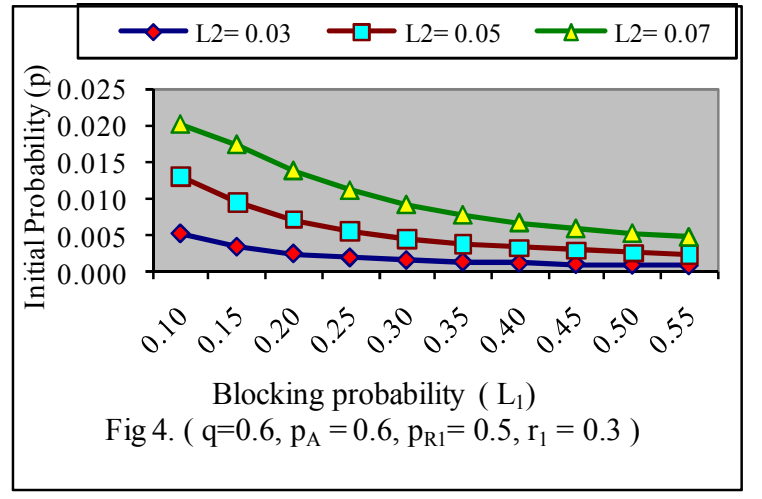

When rest state probability $p_{R I}$ is high then correspondely the traffic share of faithful users reduces for $O_{l}$. The rest state $r_{l}$ has negative impact over the group of partially impatient users (PIU).

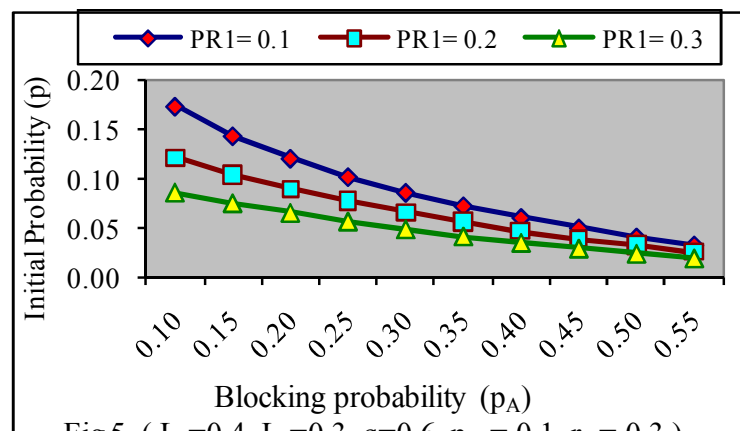

Fig 5. $\left(\mathrm{L}_{1}=0.4, \mathrm{~L}_{2}=0.3, \mathrm{q}=0.6, \mathrm{p}_{\mathrm{R} 1}=0.1, \mathrm{r}_{1}=0.3\right)$

(Between FU and PIU over varying $p_{R 1}$ )

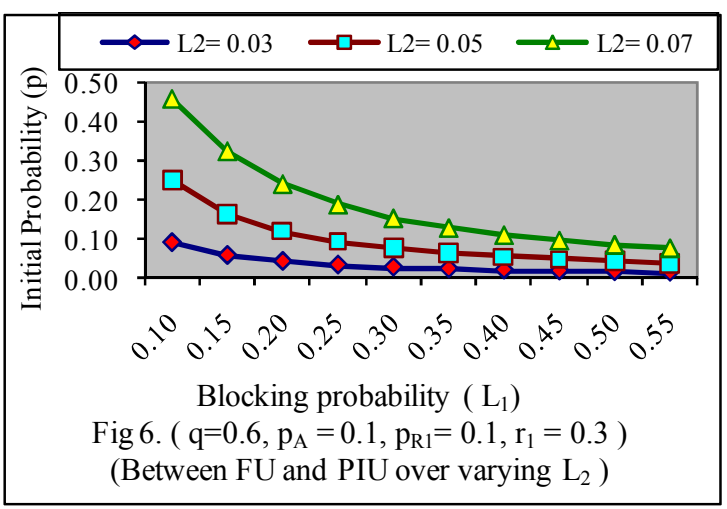

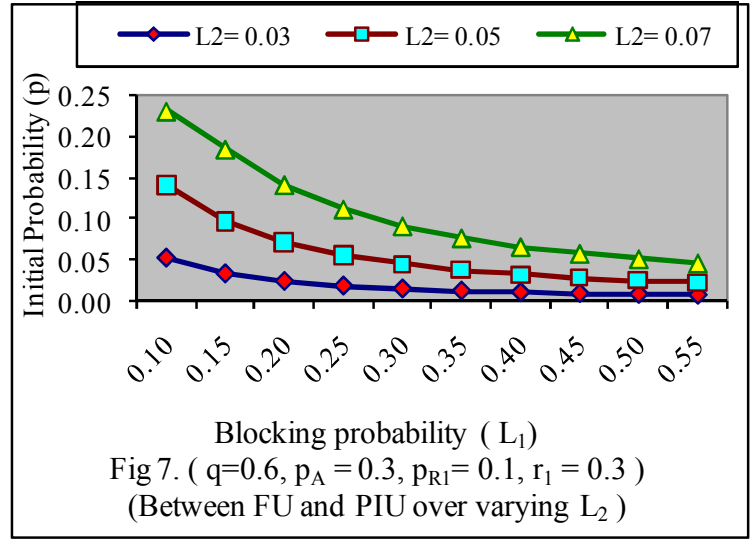

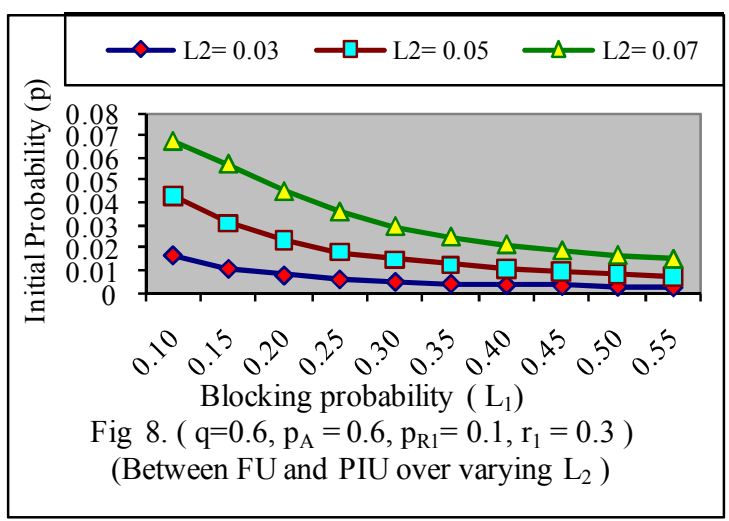

With the change of $P_{A}$ probability which is the abandoning chance if high, reduces the initial traffic share of faithful users for operator $O_{l}$. Moreover, opponent blocking level, if high, then the loss of PIU users group is also high.

\section{CONCLUDING REMARKS}

The initial traffic share depends on the amount of faithful user that an operator bears. The self blocking probability of an operator, if high, reduces the initial traffic share. Moreover, if opponent blocking of network is high, than faithful user proportion for $O_{I}$ is also high. Therefore, in multi-market system a network operator is suggested keep attracting sources and try to reduce the network blocking in order to increase his faithful user group.

\section{ACKNOWLEDGEMENTS}

Authors are grateful to the technical reviewers for the comments, which have improved the clarity content and presentation of the paper. Authors are thankful to their colleagues of the Department of Computer Science and Applications for help in many ways to complete the simulation study. 


\section{REFERENCES}

[1] Mohammed, L. A., Ramli, A. R., Daud, M. and Prakash, V., 2002, an authentication protocol for mobile cellular network, Malaysian Journal of Computer Science.

[2] Gawand, Hemangi Laxman, Murali, N. and Swaminathan, P. 2011 Presenting a new protocol for probabilistic quality of service analysis for distributed control system, International Journal of Computer Applications.

[3] Hambali, H. and Ramani, A. K., 2002 A performance study of atm multicast switch with different traffics, Malaysian Journal of Computer Science.

[4] Yeian, C. and Lygeres, J. 2005 Stabilization of a class of stochastic differential equations with markovian switching, System and Control Letters.

[5] Dorea, C.C.Y., Cruz and Rojas, J. A. 2004 Approximation results for non-homogeneous markov chains and some applications, Sankhya.

[6] Shukla, D., Gadewar, S. and Pathak, R.K. 2007 A stochastic model for space division switches in computer networks, International Journal of Applied Mathematics and Computation, Elsevier Journals.

[7] Shukla, D., Pathak, R.K. and Thakur, Sanjay 2007 Analysis of Internet traffic distribution between two markets using a markov chain model in computer networks, Proceedings of National Conference on Network Security and Management (NCSM-07).

[8] Andrikopoulos, I., et al., 1999 Providing rate guarantees for internet application traffic across ATM networks, Communications Surveys \& Tutorials, IEEE,

[9] Shukla, D. and Thakur, Sanjay, 2007 Crime based user analysis in internet traffic sharing under cyber crime, Proceedings of National Conference on Network Security and Management (NCSM-07).

[10] Perzen Emanual, 1992 Stochastic Processes, HoldenDay, Inc., San Francisco, California.

[11] Medhi, J. 1991 Stochastic models in queuing theory, Academic Press Professional, Inc., San Diego, CA.

[12] Medhi, J. 1992 Stochastic Processes, Ed.4, Wiley Eastern Limited (Fourth reprint), New Delhi.

[13] Naldi, M. 2002 Internet access traffic sharing in a multi-user environment, Computer Networks.

[14] Newby, M. and Dagg, R. 2002 Optical inspection and maintenance for stochastically deteriorating systems: average cost criteria, Jour. Ind. Statistics Associations.

[15] Mohammed. A. Al-Shargabi, Abdul Samad Ismail, and Sevia M. Idrus, 2011 Internet compressed traffic: a solution for the explosion of the internet, International Journal of Computer Applications.

[16] Aggarwal, Rinkle and Kaur, Lakhwinder 2008 On reliability analysis of fault-tolerant multistage interconnection networks, International Journal of Computer Science and Security (IJCSS).

[17] Paxson Vern, 2004 Experiences with internet traffic measurement and analysis, ICSI Center for Internet Research International Computer Science Institute and Lawrence Berkeley National Laboratory.

[18] Shukla, D., Tiwari, Virendra, Thakur, S. and Deshmukh, A. 2009 Share loss analysis of internet traffic distribution in computer networks, International Journal of Computer Science and Security (IJCSS), Malaysia.

[19] Shukla, D., Tiwari, Virendra, Thakur, S. and Tiwari, M. 2009 A comparison of methods for internet traffic sharing in computer network, International Journal of Advanced Networking and Applications (IJANA).

[20] Shukla, D., Tiwari, V. and Kareem Abdul, 2009 All comparison analysis in internet traffic sharing using markov chain model in computer networks, Georgian Electronic Scientific Journal: Computer Science and Telecommunications.

[21] Shukla, D., Tiwari, Virendra, and Thakur, S. 2010 Effects of disconnectivity analysis for congestion control in internet traffic sharing, National Conference on Research and Development Trends in ICT (RDTICT-2010), Lucknow University, Lucknow.

\section{AUTHOR'S BIOGRAPHY}

Dr. Virendra Kumar Tiwari is working as an Associate Professor in Department of Computer Science and Applications (MCA), Lakshmi Narain College of Technology (Accredited by NBA AICTE, An ISO 9001: 2000: Certified Institute), affiliated to Rajiv Gandhi Technical University (RGTU), Bhopal, M.P., INDIA and having over 10 years experience of teaching to UG and PG classes. He obtained MCA, Ph.D. (Computer Science \& Applications), degree from Department of Computer Science \& Applications, Dr. H. S. Gour Central University, Sagar (M.P.)India. He is pursuing research guide in the field of Stochastic Modeling and Computer Networking. He has authored and coauthored 20 research papers in National/International journals. His current research interest is to study the internet traffic sharing under various traffic variants.

Dr. Sanjay Thakur has completed M.C.A. and Ph.D. (CS) degree from H.S. Gour University, Sagar in 2002 and 2009 respectively. he is presently working as a Professor \& HoD in the Department of Computer Science, Lord Krishna College of Technology, Indore. He did his doctoral work in the field of Computer Networking and Internet traffic sharing. He has authored and co-authored 20 research papers in National/International journals and conference proceedings. His current research interest is Stochastic Modeling of Switching System of Computer Network and Internet Traffic Sharing Analysis. 
Dr. Diwakar Shukla is working as an Associate Professor in the Department of Mathematics and Statistics, Sagar Central University, Sagar, M.P. and having over 20 years experience of teaching to U.G. and P.G. classes. He obtained M.Sc.(Stat.), Ph.D. (Stat.), degree from Banaras Hindu University, Varanasi and served the Devi Ahilya University, Indore, M.P. as a Lecturer over nine years and obtained the degree of M. Tech. (Computer Science) from there. During Ph.D., he was junior and senior research fellow of CSIR, New Delhi by qualifying the Fellowship Examination (NET) of 1983. Till now, he has published more than 75 research papers in national and international journals and participated in over 35 seminars / conferences at national level. $\mathrm{He}$ is the recipient of MPcost Young Scientist Award, ISAS Young Scientist Medal, UGC
Career Award and UGC visiting fellow to Amerawati University, Maharashtra. He also worked as a selected Professor to the Lucknow University, Lucknow, U.P., for one year and visited abroad to Sydney (Australia) and Shanghai (China) for conference participation. He has supervised eight $\mathrm{Ph}$.D. theses in Statistics and Computer Science both; and seven students are presently enrolled for their doctoral degree under his supervision. He is member of 10 learned bodies of Statistics and Computer Science both at national level. The area of research he works for are Sampling Theory, Graph Theory, Operations Research, Stochastic Modelling, Computer Network and Operating Systems. 